\title{
EDITORIAL
}

\section{IN APPRECIATION OF GEOMICROBIOLOGY}

\author{
At a time when humans have a bigger impact on the biosphere than microorganisms, a more \\ complete understanding of the interface between geology and microbiology will be crucial.
}

The interplay between the biosphere, dominated by microorganisms, and the geosphere has profoundly shaped our planet and all life on it. From the very early stages of Earth's history to the present day, geomicrobiological processes have been at work - the introduction of oxygen into the atmosphere and the subsequent development of aerobic organisms, the extraction of nitrogen minerals from rocks and their conversion into usable molecules including proteins, the ongoing global cycling of carbon, nitrogen and sulphur, and more practical present day manifestations including the production of chemicals of practical use and the degradation of their toxic consequences. Yet despite these obvious examples of biology and geology influencing each other, immense gaps remain in our understanding of the specific forces underpinning these processes and the subsequent response of individual organisms and communities.

Take global warming as an example. Microorganisms have a huge effect on atmospheric levels of methane and carbon dioxide, the most abundant of the greenhouse gases. Although some forms of microbial life transform carbon dioxide into sugars or other organic molecules, other microorganisms synthesize the gas as a byproduct of their metabolism. And while such an understanding of the global carbon cycle lends itself to straightforward represen-

“...immense gaps

remain in our understanding of the specific

forces
underpinning
[geomicro-
biological
processes]..."

metals such as uranium, arsenic and plutonium. By transferring electrons to these metals, some forms of microbial life obtain energy for growth. The outcome of this process can, with some metals, be very beneficial the reduced metals are insoluble and are effectively removed from the food chain. Factor in the complication, however, of iron or manganese oxide and major problems can occur. These metal oxides are scavengers of toxic transition metals, radioactive metals and heavy metals. If microbial reduction of these oxides occurs, there is potential for a concentrated release of these toxic molecules and the consequences could be severe. Again, what is required is a robust knowledge of these oxides, the metabolic abilities of the microorganisms in the local environment and the nature of the interface between them.

These examples represent a fraction of the challenges and opportunities that can only be addressed through a more complete understanding of the effects that occur at the intersection of the biosphere and the geosphere. To exploit these opportunities and realize the potential of geomicrobiology, action at all levels is required. The independent, longstanding disciplines of microbiology and geology are well developed and, in isolation, will continue to mature in their own directions - they will not merge in the most productive or effective way on their own. The issues facing the successful development of geomicrobiology are the same as those confronted by any collaborative research effort that bridges diverse specialities — improved educational opportunities, development of new technologies and support infrastructure, centralized and accessible resources and, of course, adequate funding.

The appropriate support and recognition of this new field, however, promises to provide not only a model for other emerging, interdisciplinary scientific research areas but also represents an investment in answering key environmental and basic science questions that promise enormous dividends in more completely understanding the Earth's history and more accurately predicting its future. 